\title{
Image Calculations vs. Experimental Micrographs
}

\author{
Tore Niermann ${ }^{1}$
}

1. Technische Universität Berlin, Institut für Optik und Atomare Physik, Berlin, Germany.

The macroscopic electronic and mechanic properties of nanostructures, interfaces, and extended defects are determined by their atomic structure. Resolving this structure is the intention of atomic resolution microscopy. However, micrographs as acquired in modern transmission electron microscopes are not only sensitive to the structure present in the sample, but also to the imaging conditions itself. Not only obvious influences like specimen thickness, and orientation must be considered, but also the state of the instrument itself (aberrations and envelopes). Even with hardware aberration correctors, residual aberrations remain and often make a direct comparison between atomic structure and the micrographs itself impossible.

The theoretical models of the coherent interaction process between structure and the probing electron [1], as well as the imaging process itself $[2,3]$ are well established and can be found in textbooks. However, this process is described by 10.20 parameters (with additional parameters needed for image registration). We investigate strategies to find matching parameter sets for series of experimental micrographs of known structures. These parameter sets than can be used to perform atomic structure matching by means of image calculations in nearby regions of the specimen, where the structure itself unknown [4].

Experimentally, a focal-series of HRTEM images was recorded around the Gaussian focus. Focal series are less noise-limited than single exposures. Additionally, the ambiguities of non-linear image transfer are mitigated due to defocus-induced variations in the contrast transfer. The different transmission properties allow recording the full information within the frequency passband transferred by the microscope.

The parameter sets are estimated by a combination of exit-wave calculations by means of the Blochwave method, TCC approximation by means of Gaussian-Hermite quadrature, and non-linear, multivariate optimization algorithms (Figure 1). Optimized parameters include structural parameters, like specimen tilt, specimen thickness, strain and microscope parameters like initial electron flux, aberrations, and image spread. We will discuss the precision of these parameters by observing the variations across different choices of initial guesses and investigated areas.

A comparison between experimental focal series and calculation in the case of a $3.2 \mathrm{~nm}$ thick Scandium Nitrite $(\mathrm{ScN})$ crystal along <110> direction is shown in Figures 1 . The full series consists of 20 micrographs (only a subset of the series is shown here). For most focus values a good match is achieved. However, small deviations can be noticed for those focal values, which result in a weak transfer of the $\{111\}$ beams (e.g. at $-1.0 \mathrm{~nm}$ ). The contrast in these micrographs is more dependent on higher order beams, and thus is more sensitive to higher order aberrations and envelope functions. Figure 2 shows the estimated exit wave as well as the estimated linear transfer function estimated for the experimental series [5]

References: 
[1] M. de Graef, "Introduction to Conventional Transmission Electron Microscopy", Cambridge University Press, Cambridge, (2003)

[2] M. Haider et al, Microsc. Microanal. 16 (2010), p. 393.

[3] A. Thust, Physical Review Letters 102 (2009), p. 220801.

[4] G. Möbus in "High-Resolution Imaging and Spectrometry of Materials", ed. F. Ernst and M. Rühle, Springer-Verlag, Berlin, Heidelberg (2003), p. 69.

[5] The author acknowledges financial support from the Deutsche Forschungsgemeinschaft (SFB 787).

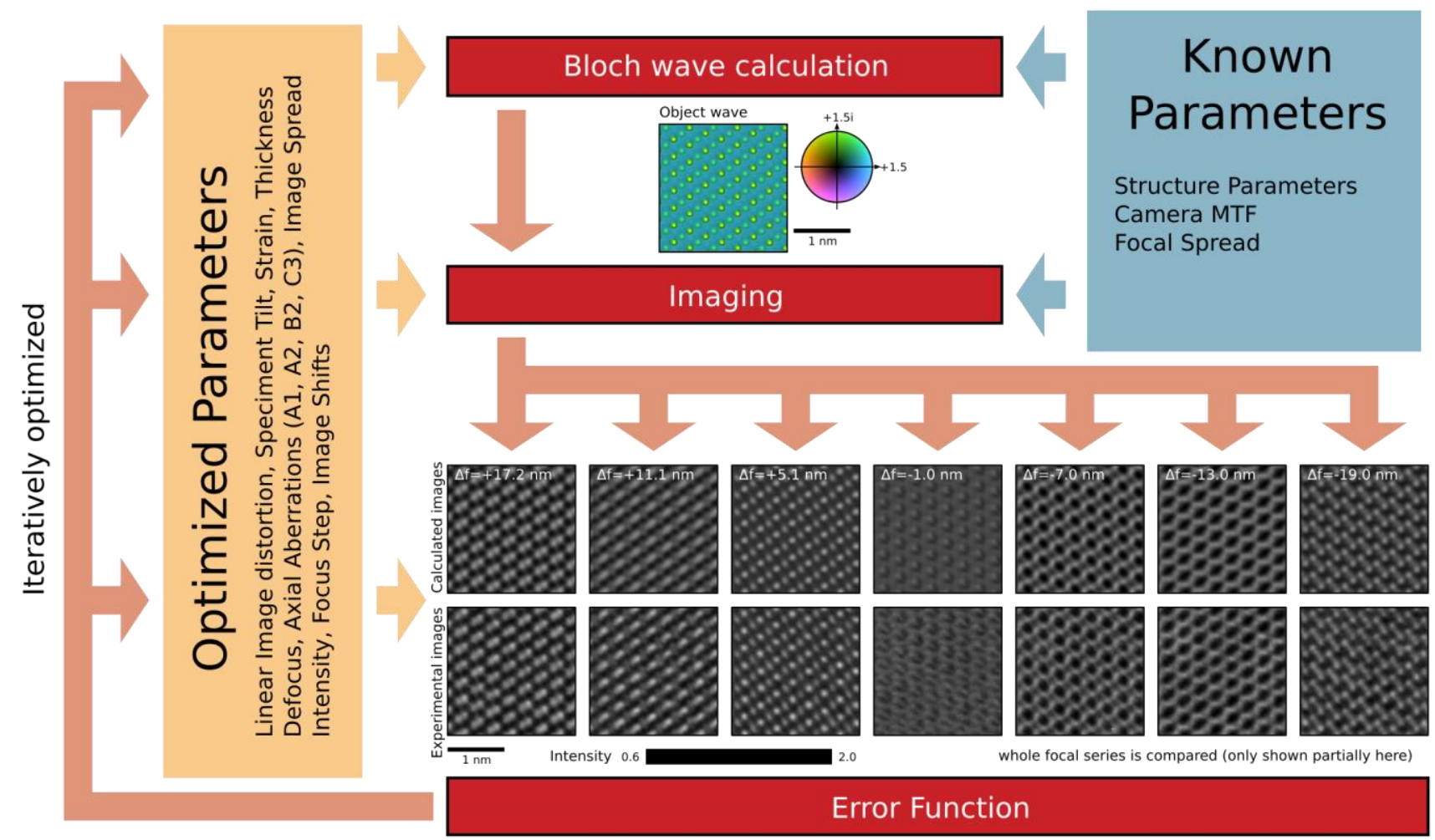

Figure 1. Optimization strategy: iterative multivariate minimization of the error function with respect to imaging parameters. In the lower right the experimental focal series of $\langle 110\rangle \mathrm{ScN}$ along the calculated images is shown.
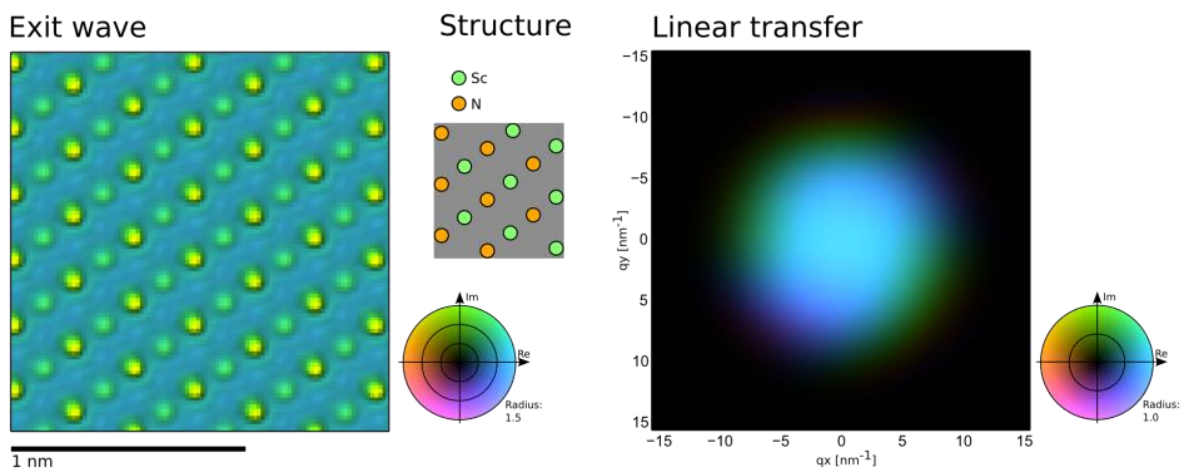

Figure 2. Left: estimated exit wave for the experimental series of Figure 1 (3.2 nm thickness, specimen is tilted by $8.4 \mathrm{mrad}$ around the vertical and by $10.9 \mathrm{mrad}$ around the horizontal). Center: A sketch of the $\mathrm{ScN}$ structure (rock-salt structure). Right: Estimated linear transfer function. 